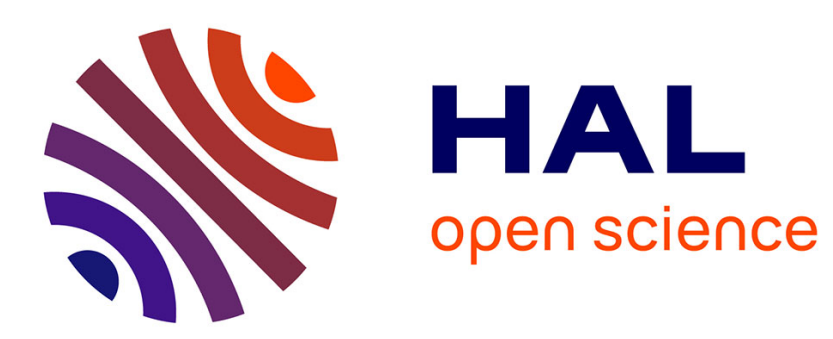

\title{
RECENT OBSERVATIONS OF THE TRANSITION TO TURBULENCE IN A PIPE
}

\author{
Tom Mullin, Jorge Peixinho
}

\section{To cite this version:}

Tom Mullin, Jorge Peixinho. RECENT OBSERVATIONS OF THE TRANSITION TO TURBULENCE IN A PIPE. Rama Govindarajan. IUTAM Symposium on Laminar-Turbulent Transition, pp.45-55, 2006, 10.1007/1-4020-4159-4_5 . hal-02143191

\section{HAL Id: hal-02143191 \\ https://hal.science/hal-02143191}

Submitted on 4 Jun 2019

HAL is a multi-disciplinary open access archive for the deposit and dissemination of scientific research documents, whether they are published or not. The documents may come from teaching and research institutions in France or abroad, or from public or private research centers.
L'archive ouverte pluridisciplinaire HAL, est destinée au dépôt et à la diffusion de documents scientifiques de niveau recherche, publiés ou non, émanant des établissements d'enseignement et de recherche français ou étrangers, des laboratoires publics ou privés. 


\title{
RECENT OBSERVATIONS IN THE TRANSITION TO TURBULENCE IN A PIPE
}

\author{
T. Mullin \& J. Peixinho \\ Manchester Centre for Nonlinear Dynamics, \\ Department of Physics and Astronomy, \\ The University of Manchester, \\ Manchester M13 9PL, UK
}

\begin{abstract}
We report the results of an experimental investigation of the transition to turbulence of Poiseuille flow in a long pipe. Our findings confirm that the recently established scaling law for the finite amplitude perturbation required to cause transition is $O\left(R e^{-1}\right)$. New results are presented concerning the decay of disturbances injected into the flow field at values of $R e$ where the flow is known to be globally stable. Exponential decay and critical behaviour is observed and these are consistent with observations in other shear flows. This new approach has enabled us to uncover a sharp cut off at the lower limit of the stability threshold.
\end{abstract}

The origins of turbulence in the flow along a circular pipe has intrigued scientists for more than one hundred and twenty years since Reynolds's [1] landmark experimental investigations. Mathematically, the flow is linearly stable [2] so that laminar flow ought to be observed for all flow rates. In practice, however, pipe flows are typically observed to be turbulent even at modest flow rates. Hence there is a direct conflict between theory and observation. Understanding this enigma has been one of the outstanding challenges of hydrodynamic stability for more than a century. Reynolds also showed that if disturbances at the inlet to the pipe are minimized, laminar flow can be maintained to higher flow rates than if they are not. Kelvin [3] proposed that finite amplitude perturbations are most likely to be responsible for triggering the transition to turbulence. The finite amplitude nature of the transition process was confirmed in the experiments of Pfenniger [4] who managed to obtain laminar flows up Reynolds numbers of $R e \approx 100,000$ by taking extraordinary care to control 
external influences ( $R e=\frac{U a}{\nu}$ where $U$ is the peak velocity, $a$ is the pipe radius and $\nu$ the kinematic viscosity of the fluid.)

The issue of transition to turbulence in pipe flow is not only of deep scientific interest, it is also of significant engineering importance. Transitional flows necessarily involve large pressure variations since the pressure gradient required to drive laminar and turbulent flows may differ by an order of magnitude. Thus flows in oil and gas pipelines are often run inefficiently turbulent to avoid the large pressure fluctuations found in the transitional regime. In addition, the control of turbulence is a dream of many practitioners, just as an understanding of turbulence is the desire of many scientists.

Linear stability of the flow means that infinitesimal disturbances added to Poiseuille flow will decay as they propagate along the pipe and laminar flow will be recovered downstream. Available experimental evidence from Reynolds and Pfenniger suggests that the influence of such disturbances is likely to become more important as $R e$, increases. A mathematical statement [5] of these facts is provided by: if $\epsilon=\epsilon(R e)$ denotes the minimal amplitude of all finite perturbations that can trigger transition, and if $\epsilon$ scales with $R e$ according to

$$
\epsilon=O\left(R e^{\gamma}\right)
$$

as $R e \rightarrow \infty$, then what is the exponent $\gamma$ ? A negative value of $\gamma$ will be consistent with the observations and one substantially less than zero would indicate that the sensitivity of the laminar flow to perturbations increases rapidly with $R e$. An outstanding problem is relating this theoretical concept to observation in a quantitative manner. Now, we review some recent experimental evidence which suggests that it is beneficial to consider the problem in this way. We will focus on the issues associated with the stability of fully developed Poiseuille flow. Hence we will not discuss the important practical problem of developing or entrance flow which can feature linear instability (see da Silva and Moss [8] for a review of this problem).

In general terms, pipe flow may be considered as a nonlinear dynamical system $d \mathbf{u} / d t=f(\mathbf{u}, R e)$ which represents the Navier Stokes equations subject to appropriate forcing and boundary conditions. The single control parameter $R e$ determines the dynamical state of the system such that there is one linearly stable fixed point, Poiseuille flow, for all $R e$ and another attractor, turbulence, when $R e>R e_{c}$. Hence when $R e<R e_{c}$ all initial conditions are attracted to the laminar state which is the global attractor for the system. When $R e \gg R e_{c}$ nearly all initial conditions give rise to turbulence so that the laminar state is now a local attractor. In practice, $R e_{c} \lesssim 1750$ so that all disturbances will decay as $t \rightarrow \infty$ for values of $R e$ smaller than this.

Experimental evidence [10] has shown that when $R e \gtrsim 1750$, small amplitude perturbations introduced into fully developed Poiseuille flow decay as 
they travel downstream i.e. when $R e \lesssim 1750$ all perturbations decay and turbulent flow cannot be maintained. On the other hand, perturbations of sufficient amplitude give rise to transition to the nontrivial state of turbulence. At these values of $R e$ the turbulence is localised and has the form of a turbulent 'puff' [6]. A finite amplitude threshold therefore exists, below which laminar flow is maintained and above which turbulence sets in. The boundary is not sharp but is probabilistic in nature [22]. Nevertheless, a definite demarcation can be established between perturbations which give rise to transition and those which do not.

A threshold curve was established by Darbyshire and Mullin [10] as a function of Re using a constant mass flux experiment with impulsive perturbations. It was found that the the amplitude of perturbation required to cause transition reduced when $R e$ was increased from 1750 and became independent of $R e$ for $R e \gtrsim 3000$. In a more recent investigation [14] a novel type of perturbation was used to uncover a scaling relationship for the amplitude of perturbation required to cause transition to turbulence. The novel feature of the perturbation is that it allowed for a separation of amplitude and timescales by injecting a boxcar distribution of perturbed fluid into the main flow field. It was firmly established that the important criterion was the length of the flow field which was perturbed and this enabled the uncovering of a $O\left(R e^{-1}\right)$ scaling law for the amplitude of perturbation required to cause transition over a wide range of Re. Some evidence for such scaling laws has been reported previously for boundary layers [23], pipe flows [7] and has also been found for plane Couette flows [20].

One surprising consequence of this finding is that the absolute amplitude of the perturbation remains relatively large with increasing $R e$. Therefore, theories based on local analyses of the trivial state may not provide much insight into transition since the basin of attraction of the laminar state remains finite even at modest $R e$. This appears to contradict many observations which show that very small amplitude disturbances are required to promote turbulence at high $R e$. However, most of these are concerned with the entrance or developing flow which is linearly unstable [8] whereas fully developed flow is not. In summary, the fully developed flow always requires a finite amplitude disturbance to cause transition.

When drawing a connection between experimental observations and theory, the difficult issue of what is meant by a perturbation must be addressed. In models, the temporal and spatial form of any perturbation can be accurately specified. On the other hand, experimentalists rely on injecting and or subtracting fluid through slits or holes in an attempt to mimic the mathematical process. The perturbation can be either periodic [7, 9] or impulsive [10] but specifying a form which can be directly related to theory is difficult. Indeed, 
identifying the part of the physical perturbation which initiates the transition process is in itself a difficult exercise although progress is being made [9]. Moreover, the robust scaling law uncovered by Hof et al. [14] also shows that self consistency can be found.

Modern theoretical research may be broadly split into two approaches. In one, initially small disturbances on the laminar state grow in a transient phase $[17,21]$ until they reach a sufficiently large amplitude so that nonlinear effects become important. These ideas have been explored for various lowdimensional models [13] and applied to plane Poiseuille flow [16, 15] and scaling laws for the amplitude of the perturbation as a function of $R e$ have been provided. An alternative point of view [5] is that the turbulent state originates from instabilities of a finite amplitude solution which is disconnected from the base state. The basin of attraction of the turbulent state grows with $R e$ so that any small perturbation will kick the laminar solution towards it. Such solutions of the Navier Stokes equations are known to exist other flows [19, 20, 18] and more recently they have been shown to exist in Poiseuille flows [24, 25]. Their lower limits of existence are almost a factor two below the range of Re where turbulence can be established but recent experimental evidence [27] suggests that they may play a role in observed turbulent structures. The stability of these new solutions and their role in transition are both currently open questions.

The $O\left(R e^{-1}\right)$ scaling law discussed above raises an interesting issue. The perturbation amplitude is normalized by the mass flux of the mean flow and thus is effectively scaled by $R e$. The dimensional perturbation amplitude is hence independent of Re which suggests that, in principle, the flow can be destabilized for any value of $R e$ provided the perturbation disturbs sufficient length of the flow field. It is known that the flow is globally stable for $R e \lesssim 1750$ and hence there is inconsistency between these new results [14] and well established facts. Here we report the observations of a new experimental investigation where we study the processes whereby injected perturbations decay for $R e \lesssim 1750$ and show that there is a surprisingly sharp cut-off in the threshold curve.

\section{Experimental details}

The experimental system can be regarded as a large hypodermic syringe where a piston pulls water at a fixed mass flux along a 15.7 metre 'needle'. A schematic diagram of the apparatus is shown in figure 1. The pipe consisted of a $D=20 \pm 0.01 \mathrm{~mm}$ diameter Perspex tube which was constructed using $150 \mathrm{~mm}$ long machined sections push-fitted together and butted flush so that there was no measurable gap between each join. The sections were held on 
on a steel base with a total length of $15.7 \mathrm{~m}(785 \mathrm{D})$ and were aligned using a laser.

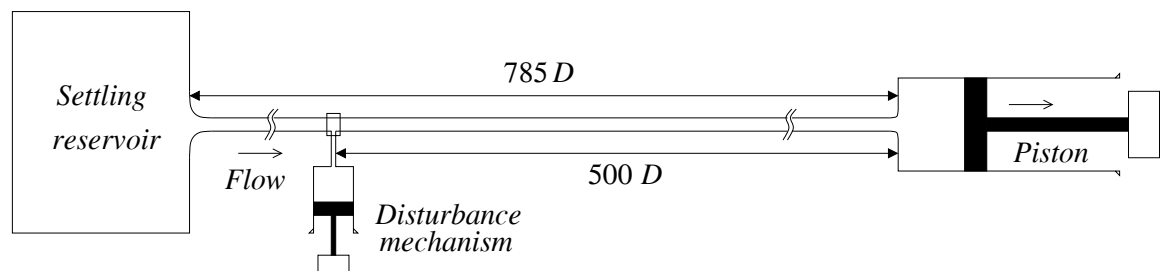

Figure 1. Schematic of the constant mass flux pipe facility

A reservoir with a capacity of approximately 100 liters was connected to the pipe entrance via a smooth trumpet shaped inlet. This device ensures a laminar flow over the whole $R e$ range investigated for a flow which was initially disturbance free. The maximum flow rate achievable corresponded to $R e=23,000$ and laminar flow could be achieved with care. On the other hand a sharp cornered inlet induced transition spontaneously at a $R e \approx 2000$.

The fluid was pulled through the pipe by a cylindrical machined steel piston of length $1.033 \mathrm{~m}$ and $0.260 \mathrm{~m}$ diameter. The expansion aspect ratio between the piston and the pipe was 13 and a smooth trumpet joint was again used. The piston was pulled by a lead screw and nut arrangement. This was powered by d.c. motor which was computer controlled allowing the speed to be varied as a function of time to within an accuracy of $1 \%$. Hence, even if the fluid in the pipe became turbulent, the mass flux pulled through the pipe was unaffected so that the Re remained constant.

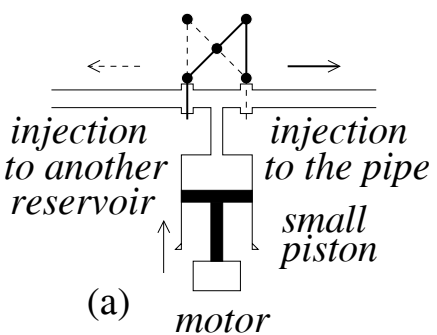

(a) motor

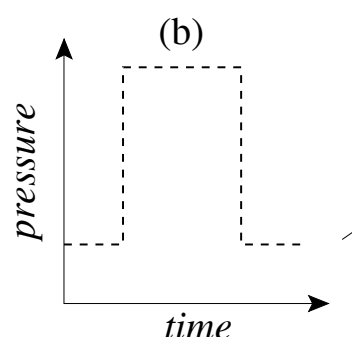

time (c)

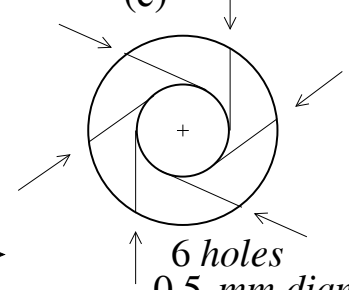

$0.5 \mathrm{~mm}$ diameter

Figure 2. (a) Schematic of the disturbance generator. (b) Typical trace of the perturbation. (c) Inlet manifold of six jet disturbance 
The long term temperature stability of the laboratory was set at $20 \pm 1{ }^{\circ} \mathrm{C}$ using several air-conditioning units which were located adjacent to the pipe. The largest temperature gradient recorded from several $K$-type thermocouple along the pipe was $0.3^{\circ} \mathrm{C}$ which corresponded to a variation in $R e$ of \pm 30 at $R e=2000$.

Two types of experiments were performed. In the first, fully developed laminar flow [12] was established over the $R e$ range investigated and the stability of the flow was probed using a perturbation which was applied at 285 pipe diameters from the pipe entrance. When turbulent flow (in the form of puffs,slugs etc) was observed at the end of the pipe transition was deemed to have occurred. In the second, a well defined perturbation was injected into fully developed flow and the evolution of the disturbance was observed as it progressed downstream.

In both sets of experiments the perturbation was provided by a single boxcar pulse of fluid which was injected tangentially into the flow via a ring of six equally spaced $0.5 \mathrm{~mm}$ holes as shown in figure 2(c). The valves which control the fluid injection had switching times of approximately $1 \mathrm{~ms}$ are shown schematically in figure 2(a). A typical pressure trace of the perturbation is shown figure 2(b). The injection system enabled variation of both the duration $(0<\Delta t<10 \mathrm{~s})$ and the amplitude $\left(0<\Phi_{i n j}<5 \mathrm{ml} / \mathrm{s}\right)$ of the perturbation. The displaced volume $\Phi_{i n j}$ from the injector is used in our definition of the amplitude $A$ of the perturbation. The quantities of fluid injected can be the range 0.0001 to $0.2 \%$ of the total mass flux where the larger values were required to cause transition at smaller $R e$. The duration of the injection set the spatial extent of the disturbed flow ( $L^{*}=\Delta t * \bar{U}$ in pipe diameters) since it initially travel with the mean speed $\bar{U}$ of the flow in the pipe. The relative volume flux $\Phi_{i n j} / \Phi_{\text {pipe }}$ is used to define the amplitude of the perturbation $A$. In principle, the perturbation will affect the flow field globally but previous tests using both injection and suction [10] showed that it is localized in practice.

The flow state was monitored using flow visualization where a small amount of Mearlmaid Pearlessence material was added to the water. The suspended particles were anisotropic in shape and had the form of $\sim 35 \times 10 \mu \mathrm{m}$ platelets. They reflected the incident light from a $\sim 3 \mathrm{~mm}$ vertical light-sheet which was formed all along the pipe. The light-sheet was switched on and off sequentially to reduce heating effects. A photograph of a typical turbulent puff is given in figure 3 .

The design of the experiment was such that it ran in single shot mode. Transients at both the beginning and the end of each run were approximately $10 \mathrm{~s}$ long and these were independent of $R e$. A typical useful experimental time was half an hour at $R e=2000$. After each run of the experiment, the fluid was pushed back through the pipe into the reservoir and allowed to settle for a period of at least 30 minutes before the next run was started. This period was 


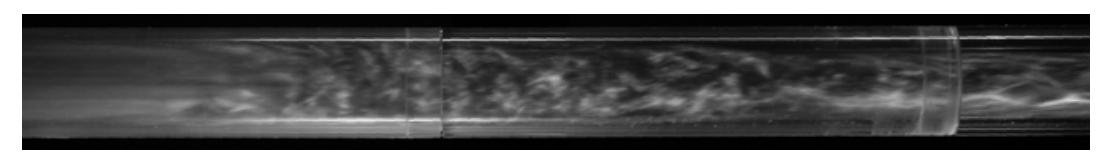

Figure 3. A typical puff at $R e=1900$

chosen empirically on the basis of observations of the fluid in the tank and it was also found to be the minimum time required to give repeatable results.

In the second set of experiments on the evolution of disturbances, it occasionally proved possible to obtain results from several sequential experimental runs. This helped improve the statistics of the measured distributions.

\section{Results}

\section{Finite Amplitude Stability Curve}

As discussed in the introduction, Hof et al. [14] developed an injection system which permits the amplitude and width of the perturbation to be varied independently. This helped uncover a scaling law which indicates that the amplitude of perturbation required to cause transition scales as $O\left(R e^{-1}\right)$. One interpretation of this result is that it reflects the balance between viscous and inertia terms in the Navier Stokes equations. The amplitude of perturbation required to cause transition was smallest when $L^{*} \gtrsim 6 D$ and independent of $R e$ for values of $L^{*}$ greater than this. All of the new estimates for the stability threshold have been obtained with $L^{*} \gtrsim 6 D$. We have added these new estimates to the previous results and a compilation of both sets is presented in figure 4(a). In general, there is very good agreement between both sets and a $O\left(R e^{-1}\right)$ scaling law for the amplitude of perturbation required to cause transition has been confirmed.

The amplitude $A$ of the perturbation in figure 4(a) is made non-dimensional by dividing by the mass flux of the main flow. Hence it is effectively scaled by $R e$. If the data is now plotted in dimensional form as in figure 4(b) it can be seen that $\Phi_{i n j}$ is almost independent of $R e$ and only departs from a constant level for $R e \lesssim 3000$. In fact, the departure is not as great as one might expect. All experimental evidence suggests that the flow is globally stable for $R e \lesssim 1750$ so that one might anticipate that $\Phi_{i n j} \rightarrow \infty$ rapidly when $R e<1750$. In practice, injecting large amplitude disturbances gave rise to wide variations in estimates of the critical points and so a new strategy was adopted. This was based on the observation that most disturbances injected 

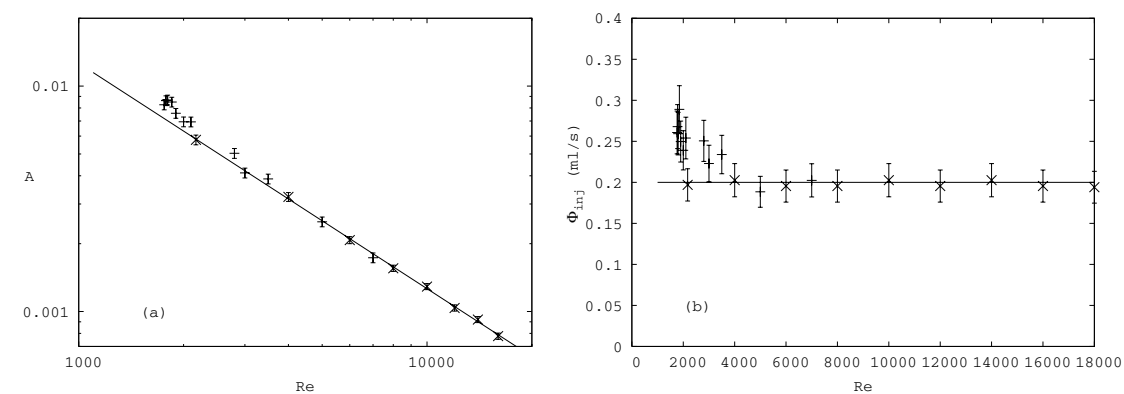

Figure 4. Plots of the stability threshold $A$ versus Re. Laminar flow was found below the line and turbulence above when perturbations of the given amplitudes were injected. (a) Log/log plot where the slope of the fitted line is -1 . (b) Dimensional plot using the same data as in (a).

into the flow when $R e<1750$ decayed as they moved downstream. It is this process that we will focus on in the next section.

\section{Decay of Injected Disturbances}

The investigation was carried out by injecting well defined perturbations into fully developed Poiseuille flow (285D from the entrance) and observing their development downstream. The values of Re investigated were such that the final state far downstream was, typically, laminar flow. At a given $R e$, the disturbed flow was localized and traveled along close to the mean speed of the flow. In the first $100 D$ the perturbation evolved in a complicated way as discussed by Wygnanski and Champagne [6] and Darbyshire and Mullin [10]. Several light boxes suspended above the pipe provided a light sheet along the length of the pipe and these were switched on and off sequentially to avoid heating effects. This illumination allowed the observation of a patch of disordered fluid as it traveled along and enabled an estimate to be made of position at which the it decayed (measured in diameters $D$ from the perturbation input).

The results presented in figure 5 are graphs of the probability of observing a localized disturbed region of flow, plotted as a function of distance downstream in $D$ from the point of injection (zero on the abscissa). The downstream limit was set by the length of the pipe at $500 D$ but this was not a severe limitation since not many disturbances survived to this station in practice. The initial conditions for the perturbation were $L^{*}=10$ and the amplitudes used in figure 5(a) and 5(b) were $A=0.01$ and $A=0.1$ respectively. Between 40 and 100 independent experimental runs were performed for each value of $R e$ in order to obtain good statistics. The straight lines correspond to least squares fits of exponentials $P(D) \propto \exp (-\epsilon D)$, where $\epsilon$ the rate of decay of the 

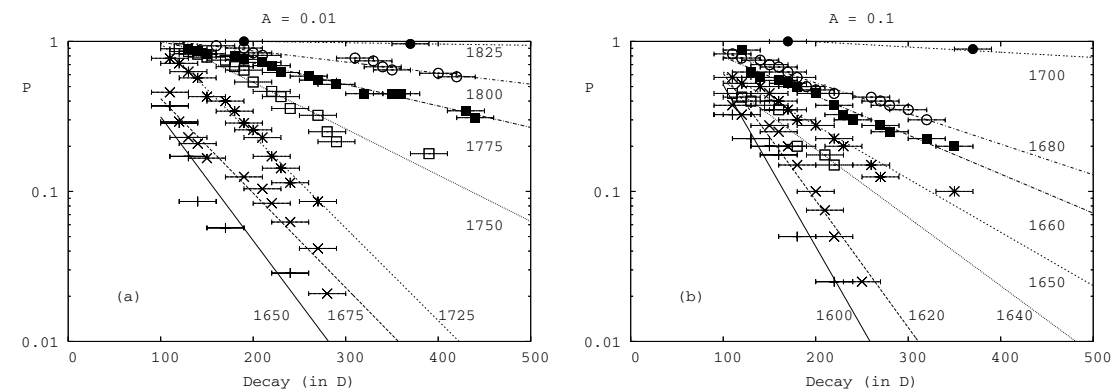

Figure 5. The probability of observing a localized region of disturbed flow plotted as a function of distance downstream from the point of injection. Data was accumulated from between 40 to 100 measurements for each of $R e$. The lines are least squares fits of exponentials. (a) The initial disturbance amplitude was $A=0.01$ (b) The initial disturbance amplitude was $A=0.1$

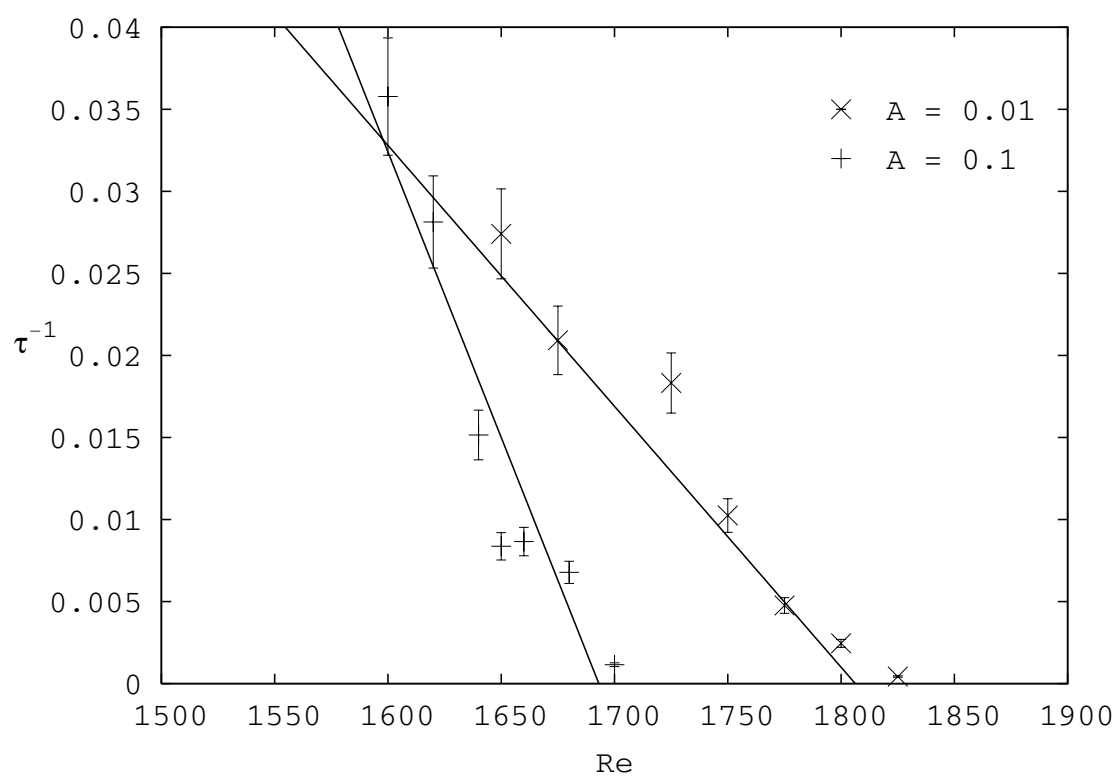

Figure 6. Half-life $\tau$ plotted as a function of $R e$ for $A=0.01$ and $A=0.1$. Estimates of $R e_{c}$ are $1830 \pm 10$ and $1710 \pm 10$ respectively

disturbed state. The quality of the fits indicate that the disturbed flow decays exponentially to a reasonable approximation. In general, the slopes increase as $R e$ decreases i.e. there is faster decay at smaller Re. Such behaviour has previously been observed by Bottin and Chate [11] in experiments on plane Couette flow. 

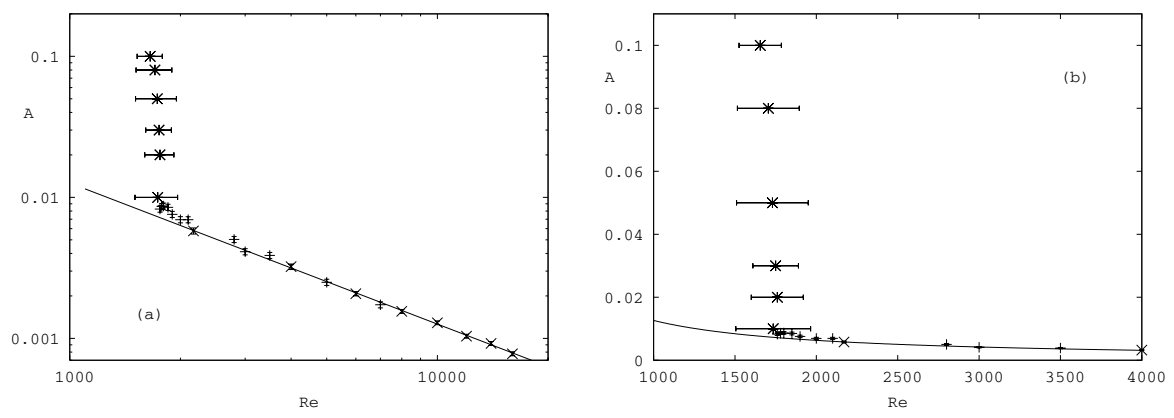

Figure 7. Finite amplitude threshold curve $A$ vs $R e$ with data from transient experiments included. (a) Log scale (b) Linear scale.

A useful measure that can be extracted from the exponential fits is time required for half the initial states to decay which is defined as $\tau=(\ln 2) / \epsilon$. We will refer to this as the 'half-life' of a perturbation. Plots of the inverse of halflife $\tau^{-1}$ versus $R e$ are shown in figure 6 for the two perturbation amplitudes used in the present series of experiments viz. $A=0.01$ and $A=0.1$. It may be seen that $\tau^{-1}$ passes through zero at $1830 \pm 10$ and $1710 \pm 10$ respectively. At these critical values of $R e$ the half-life $\tau$ approaches infinity and the perturbation does not decay but develops into a turbulent puff which persists. Hence this gives a method for estimating the threshold for transition to turbulence which is more accurate than the direct method of increasing the amplitude of perturbation until a threshold is crossed as in the results section labeled (Finite Amplitude Stability Curve) above.

This new method was used to obtain estimates of the stability threshold for $R e \lesssim 1750$ by setting the amplitude of the perturbation at a prescribed value and observing transient behaviour for a range of $R e$ as above. The new results for the estimate of the threshold curve are presented together with the previous ones in Figure 7. We have again chosen to show the results on both logarithmic (Figure 7(a)) and linear (Figure 7(b)) scales to emphasize different aspects. There is clear consistency between the two sets of results which indicates that both methods are valid ways of estimating the stability boundary. The new estimates show clearly that the stability boundary rises almost vertically for $R e \lesssim 1750$ i.e. there is a sharp departure from the $O\left(R e^{-1}\right)$ scaling law. Hence maintaining disordered flow below $R e \lesssim 1750$ is not possible since perturbations over a wide range of amplitudes decay. 


\section{Conclusions}

We have confirmed the previous results for the $O\left(R e^{-1}\right)$ scaling law for the amplitude of the perturbation required to cause transition in circular Poiseuille flow over a range of $R e$. Moreover we have extended the results using the transient decay of perturbations towards the lower values of $R e$. These new findings have uncovered a surprisingly sharp cut off in $R e$ below which turbulence cannot be maintained. It has been suggested [26] that such behaviour is consistent with the formation of a chaotic repellor and saddle in the solution set but much more work is required before definite conclusions in this respect can be made.

\section{Acknowledgements}

The authors are grateful to EPSRC for supporting this research. TM is supported by a 'Senior Fellowship' and JP by the research grant GR/576137/01.

\section{References}

[1] Reynolds O. 1883 An experimental investigation of the circumstances which determine whether the motion of water shall be direct or sinuous and of the law of resistance in parallel channels. Proc. Roy. Soc. A 35, 84-99.

[2] Drazin, P. G. \& Reid, W. H. 1980 Hydrodynamic stability, Cambridge University Press.

[3] Kelvin, Lord 1887 On the stability of steady and of periodical fluid motion, Phil. Mag. 23, 459-464.

[4] Pfenniger W. 1961 Transition in the inlet length of tubes at high Reynolds numbers. In Boundary Layer and Flow Control (ed. G.V. Lachman), 970-980, Pergamon.

[5] Waleffe, F. 1997 On a self-sustaining process in shear flows. Phys. Fluids 9, 883-900.

[6] Wygnanski, I. J. \& Champagne, F. H. 1973 On transition in a pipe. Part 1. The origin of puffs and slugs and the flow in a turbulent slug. J. Fluid Mech. 59, 281-351.

[7] Draad, A. A., Kuiken, G. D. C. \& Nieuwstadt, F. T. M 1998 Laminar-turbulent transition in pipe flow for Newtonian and non-Newtonian fluids. J. Fluid Mech. 377, 267-312.

[8] da Silva, D. F. \& Moss, E. A. 1994 The stability of pipe entrance flows subjected to axisymetric disturbances. J. of Fluids Eng. 116, 61-65.

[9] Han, G., Tumin, A. \& Wygnanski, I. J. 2000 Laminar-turbulent transition in Poiseuille pipe flow subjected to periodic perturbation emanating from the wall. Part 2. Late stage of transition. J. Fluid Mech. 419, 1-27.

[10] Darbyshire, A. G. \& Mullin, T. 1995 Transition to turbulence in constant-mass-flux pipe flow. J. Fluid Mech. 289, 83-114.

[11] Bottin, S. \& Chate, H. 1998 Statistical analysis of the transition to turbulence in plane Couette flow. Eur. Phys. J. B 6, 143-155.

[12] Fargie, D. \& Martin, B. W. 1971 Developing laminar flow in a pipe of circular crosssection. Proc. Roy. Soc. A 321, 461-476. 
[13] Baggett, J. S. \& Trefethen, L. N. 1997 Low-dimensional models of subcritical transition to turbulence, Physics of Fluids 9, 1043-1053.

[14] Hof, B., Juel, A. \& Mullin, T. 2003 Scaling of the turbulence transition threshold in a pipe. Phys. Rev. Lett. 91, art. 244502.

[15] Chapman, S. J. 2002 Subcritical transition in channel flows, J. Fluid Mech. 451, 35-97.

[16] Henningson, D. S., Lundbladh, A. \& Johansson A. V. 1993 A mechanism for bypass transition from localized disturbances in wall-bounded shear flows Henningson, J. Fluid Mech. 250, 169-207.

[17] Trefethen, L. N., Trefethen, A. E., Reddy, S. C. \& Driscoll, T. A. 1993 Hydrodynamic stability without eigenvalues, Science 261, 578-583.

[18] Anson, D. K., Mullin, T. \& Cliffe, K. A. 1989 A numerical and experimental investigation of a new solution in the Taylor vortex problem. J. Fluid Mech. 207, 475-489.

[19] Barnes, D. R. \& Kerswell, R. R. 2000 New results in rotating Hagen-Poiseuille flow. J. Fluid Mech. 417, 103-126.

[20] Dauchot, O. \& Daviaud, F. 1995 Finite amplitude perturbation and spots growth mechanism in plane Couette flow. Phys. Fluids 7, 335-343.

[21] Grossmann, S. 2000 The onset of shear flow turbulence. Rev. Mod. Phys. 72, 603-618.

[22] Eckhardt, B. and Mersmann, A. 1999 Transition to turbulence in a shear flow. Phys. Rev. E 60 509-517.

[23] Govindarajan, R. \& Narasimha, R. 1991 The role of residual nonturbulent disturbances on transition onset in two-dimensional boundary layers. J. Fluids Eng. 113, 147-149.

[24] Wedin, H. \& Kerswell, R. R. 2004 Exact coherent structures in pipe flow: Travelling wave solutions. J. Fluid Mech. 508 333-371.

[25] Faisst, H. \& Eckhardt, B. 2003 Travelling waves in pipe flow. Phys. Rev. Lett. 91, art. 224502.

[26] Faisst, H. \& Eckhardt, B. 2004 Sensitive dependence on initial conditions in transition to turbulence in pipe flow. J. Fluid Mech. 504, 343-352.

[27] Hof, B., van Doorne, C. W. H., Westerweel, J., Nieuwstadt, F. T. M., Faisst, H., Eckhardt, B., Wedin, H., Kerswell, R. R. \& Waleffe, F. 2004 Experimental observation of nonlinear travelling waves in turbulent pipe flow. Science 305, 1594-1597. 